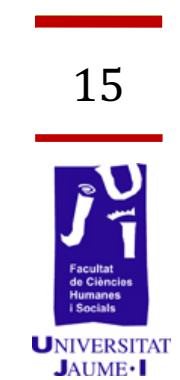

\title{
Formas de violencia hacia las mujeres en la política: repensando la práctica partidista
}


Uno de los principales motivos para preparar esta comunicación es intentar reflexionar desde la teoría, sobre lo que está pasando con las mujeres en el seno de los partidos o movimientos políticos. Hasta la actualidad, persisten formas "sutiles», y "camufladas», al interior de estas organizaciones que están afectando al derecho de participación política de las mujeres. Para ello, a breves pinceladas, partiremos de un repaso sobre la trascendencia de estas organizaciones para la democracia representativa actual. Luego pasaremos sobre algunos posicionamientos críticos, estudios y propuestas frente al problema de la violencia «micro» hacia las mujeres en el contexto de la organización interna de los partidos.

Palabras Claves: Partidos políticos; democracia; derecho de participación política; micromachismos; igualdad efectiva.

\section{Introducción}

Los partidos políticos cumplen un papel fundamental en la democracia y en el sistema político representativo contemporáneo. Facilitan que los sujetos políticos primarios, esto es: ciudadanos y ciudadanas, puedan ejercer su derecho de asociación y participación política como lo establece el art. 23 de la Constitución Española (en adelante CE). Por tanto, la participación política en el marco de los partidos debe organizarse de acuerdo con los parámetros Constitucionales de igualdad, libertad, dignidad, democracia, etc., del art. 6 CE.

Por otra parte, una las finalidades fundamentales de estas organizaciones, es la de alcanzar el poder del Estado y del Gobierno. Por lo que, mediante instrumentos electorales, políticos, de planificación interna, se proyectan constantemente al cometido de este fin. Estos se constituyen en un sujeto político de tipo colectivo, en el que nadie, con las salvedades que la ley establece, debería ser excluido de formar parte de manera libre e igualitaria.

En ese sentido, como se lo manifestó en la Conferencia de Naciones Unidas de Beijing en 1995 y en la Declaración de Atenas en 1992, las mujeres no somos un colectivo más de la pluralidad social y política que deben encarnar los partidos, sino que somos más del $50 \%$ por ciento de la voluntad del soberano, del pueblo. Por consiguiente, estas organizaciones al tener la enorme responsabilidad de plasmar y actuar y organizarse en cumplimiento con los máximos valores constitucionales, y la configuración de aquella voluntad general que legitima la democracia y las instituciones, tienen la responsabilidad política y jurídica de respetar la igualdad en su organización y funcionamiento, sin discriminación hacia hombres y mujeres. Lo que requiere que estos tengan en cuenta las realidades y problemas de ambos sexos en las diferentes escalas de adhesión, esto es: como afiliados o afiliadas, militantes, dirigentes, simpatizantes, etc., y en 
todo el itinerario de participación política que no solo se limita en el diseño y conformación de listas, sino que empieza desde el momento de la adhesión, afiliación, procesos internos de organización, toma de decisiones, y durante el desempeño del cargo para el que fueron electas en ejercicio del derecho a ser elegidas.

\section{Objetivos}

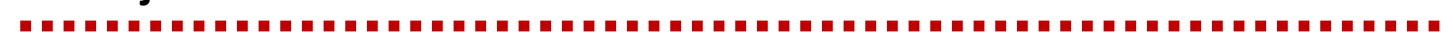

Este artículo, tiene como objetivo: reflexionar sobre los roles de género presentes en los partidos políticos con especial atención en las actitudes machistas en sus diferentes intensidades. El tema de esta comunicación se ubica, por una parte, en el ámbito de lo que Falco $\mathrm{LANCHESTER}^{1}$ denomina como de «legislación electoral de contorno», que se refiere a todo lo relacionado con el acceso igualitario a la competición electoral, a las garantías de dicha igualdad de oportunidades entre los sujetos participantes en dicha competición, y a la participación política no electoral dentro de los partidos.

\section{Discusión}

Para asegurar la igualdad de género al interior de los partidos, es necesario reconocer que la mitad del soberano: es decir las mujeres, ejercen sus derechos políticos pasivos o activos, con un doble esfuerzo en relación con los hombres, y en medio de lo que algunas autoras ${ }^{2}$ definen como sistema de género. Donde se infravalora la presencia política de las mujeres en la esfera pública mediante la división política-sexual de los roles y funciones políticas al interior de los partidos.

Sin embargo, hablar de este sistema en su versión política, en todos sus componentes, no es suficiente, para eso es necesario reconocer las diferentes intensidades y escalas en las que se desarrolla dicho sistema. Lo que significa reconocer la existencia de un paradigma masculino hegemónico, donde los hombres mediante sus opiniones, perfiles, valores, actitudes y percepciones, siempre han sido el referente. Esto no nace de la nada, el paradigma del ahora es el producto de un paradigma presente desde siempre, especialmente ratificado con el surgimiento de la modernidad desde las teorías contractualistas, como lo explica ROSA COBO en su libro sobre los Fundamentos del Patriarcado Moderno ${ }^{3}$.

\footnotetext{
${ }^{1}$ LANCHESTER, F. «La propaganda elettorale (e referendaria) in Italia tra continuità sregolata e difficile rinnovamento», Quad. Cost., año XVI, nro. 3, 1996, p. 387.

${ }^{2}$ Para ampliar la referencia, Véase en: ASTELARRA, Judith. Veinte años de políticas de igualdad. Ediciones Catedra. Madrid, 2005. p. 18. COBO, Rosa. Fundamentos del patriarcado moderno: Jean Jacques Rousseau. Ediciones Catedra. Madrid, 1995. p. 261. FIGUERUELO BURRIEZA, Ángela. "Representación política, derecho de asociación, y democracia Paritaria", Curso de Verano: Nuevas Tecnologías, administración y participación Ciudadana, Universidad de Salamanca, 1 de Julio de 2007. www.letrasjuridicas.com/Volúmenes/18/figueruelo18.pdf. p. 12.

${ }^{3} \mathrm{COBO}$, Rosa. Fundamentos del patriarcado...lbídem.
} 
Para Luis BONINO, dicho paradigma masculino hegemónico en sus diferentes escalas, nos permite hablar de machismo de alta intensidad, de baja intensidad o también llamados micromachismos. Para hacer una mejor diferenciación de esta escala, diremos que, los primeros tipos de machismos son los tradicionalmente proscritos por la ley, en contraste con los micromachismos que muchas veces son aceptados y pasan desapercibidos. En su gran mayoría, aparecen y se cometen por discriminación indirecta, donde se produce un tratamiento formalmente neutro, resultan mucho más difíciles de detectar ya que solo puede medirse por sus efectos, donde incluso muchas veces llegan a encontrar justificación política.

El autor los define como: "...las actitudes de dominación "suave» o de "bajísima intensidad», formas y modos larvados y negados de abuso e imposición en la vida cotidiana. Son, específicamente, hábiles artes de dominio, comportamientos sutiles o insidiosos, reiterativos y casi invisibles que los varones ejecutan permanentemente. Estos toman fuerza cuando se posicionan en un contexto en que las violencias y dominaciones masculinas más explicitas y de más alta intensidad, se están deslegitimando socialmente cada vez más, en su lugar operan estos micromachismos en el imaginario político como armas, trucos, tretas y trampas frecuentes y sutiles de algunos partidos y políticos, que sirven para ejercer e imponer su «autoridad» sobre las mujeres, mediante un repertorio de comportamientos considerados «normales». No necesariamente suponen intencionalidad, mala voluntad, ni planificación deliberada, sino que son dispositivos mentales y corporales automatizados en el proceso de "hacerse hombres», como hábitos de funcionamiento frente a las mujeres. Otros en cambio sí son conscientes, porque de una u otra forma son parte del repertorio masculino, de sus modos de estar y afirmarse en el mundo, buscan disminuir la libertad de elegir y decidir de las mujeres. ${ }^{4}$

Estos comportamientos hacia las mujeres se desarrollan en el contexto de una PIRAMIDE DE PODER dentro de los partidos. Que según un estudio realizado por el Instituto Internacional para la Democracia y la Asistencia Electoral (en adelante IDEA Internacional) el Banco Interamericano de Desarrollo (en adelante BID) en el 2010 sobre partidos y paridad, explican la poca presencia de las mujeres en los espacios de poder, aunque constituyan cerca o más del $50 \%$ de la militancia y afiliados o afiliadas. Es decir, su presencia es mayoritaria en espacios de menos jerarquía lo que va mermando hasta ser mínimo en los espacios de máxima representación. Así lo vemos a continuación.

\footnotetext{
${ }^{4}$ Véase en BONINO, Luis. "Los micromachismos». Revista La Cibeles No 2. Ayuntamiento de Madrid, noviembre 2004. pp. 2 y ss.
} 

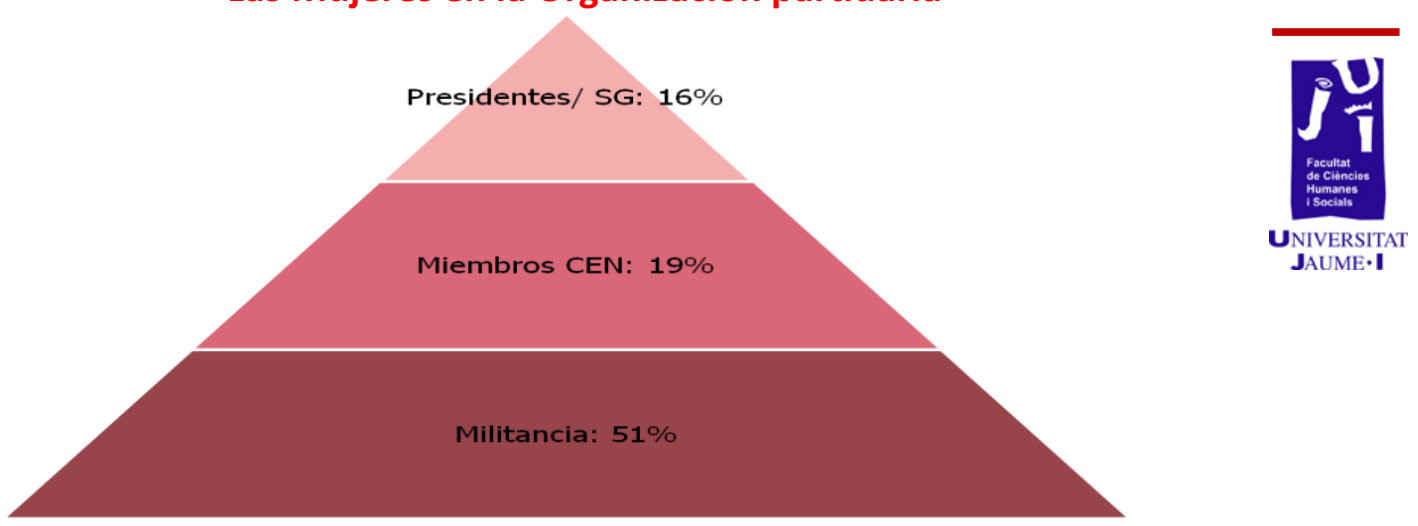

Fuente: ROZA, Vivian (2010). Et. Al. Partidos políticos y paridad: La ecuación pendiente. Publicaciones IDEA internacional: Suecia, New york. p. 28.

En otro estudio diagnóstico realizado por la Asociación de Concejalas de Bolivia (en adelante ACOBOL), que sirvió de base para la presentación de un proyecto de ley al respecto. Los comportamientos más usuales que evidencian la violencia contra las mujeres al interior de los partidos políticos se caracterizan porque en su gran mayoría toman la forma de micromachismos, aunque muchas veces con connotaciones de machismos de alta intensidad. Por tanto, entre los mecanismos indirectos de discriminación encontrados, están:

$\checkmark$ La utilización sexista del lenguaje

$\checkmark$ Requerimientos innecesarios para el desempeño del puesto que se apoyan en estereotipos

$\checkmark$ La nula comunicación o trasmisión de información falsa,

$\checkmark$ El establecimiento de requisitos que resulten de más difícil cumplimiento para las mujeres que para los hombres: horarios, movilidad, etc.

$\checkmark$ La imposición por razón de género de la realización de actividades y tareas ajenas a las funciones y atribuciones generales de toda autoridad.

$\checkmark$ El establecimiento de un nivel retributivo inferior a la categoría profesional desempeñada por mujeres.

$\checkmark$ Las utilización y abuso de la justicia comunitaria en contra de las autoridades elegidas para un cargo público, etc. ${ }^{5}$

La Asociación de Mujeres Municipalistas de Ecuador, (en adelante AMUME), sostiene que entre los principales rasgos de este tipo de violencia machista, están:

$\checkmark$ Injerencias arbitrarias y abusivas en el desempeño de sus funciones.

$\checkmark$ Desprestigio, descalificación, estigmatización, a veces incluso con connotaciones sexuales.

$\checkmark$ Manipulación, asedio y hostigamiento.

\footnotetext{
${ }^{5}$ ROJAS, María Eugenia. Acoso y Violencia política en contra de Mujeres Autoridades públicas electas
} en los gobiernos locales-municipales en Bolivia. ONU-Mujeres, ACOBOL, Bolivia, 2012. 
$\checkmark$ Disminución, impedimento o restricción en el ejercicio del cargo para el cual ha sido electa y las posibilidades de presentarse como candidata a procesos de elección o reelección.

$\checkmark$ Coincidiendo con el informe de ACOBOL, también se produce la negación de información, que incluye no informar sobre la realización de las sesiones del Concejo o asamblea, impidiéndoles participar en los procesos de toma de decisiones, y ocultamiento o violación de correspondencia.

$\checkmark$ Interferencias en la comunicación con organizaciones y ciudadanía.

$\checkmark$ Negación o retardo en el pago de dietas que les corresponden en derecho.

$\checkmark$ Presión individual y/o colectiva para que las mujeres asuman las decisiones que convienen a determinados intereses, y para que actúen en contra de su voluntad.

$\checkmark$ Presión para que cedan espacios de representación ante otras instancias,

$\checkmark$ Denegación de recursos económicos necesarios para asumir representaciones.

$\checkmark$ Desestimación, ridiculización y descalificación pública de las propuestas presentadas por las mujeres, especialmente cuando éstas están relacionadas con la promoción de los derechos de las mismas.

$\checkmark$ Negación de la participación de las mujeres en las sesiones, obligándolas sólo a firmar actas, aun cuando no hubieran participado.

$\checkmark$ Agresiones verbales y generación de un ambiente hostil a las mujeres, especialmente cuando éstas son minoría en concejos o asambleas.

$\checkmark$ Daños en la gestión de mujeres autoridades locales ${ }^{6}$.

Cuando hace un momento me referida a que, no hay mejor espacio de cultivo para los micromachismos que la discriminación indirecta, me refería a que muchos de estos comportamientos encuentran «cierta legitimación» en argumentos o justificaciones políticas que se suponen "naturales», «neutrales» y tradicionalmente aceptados por el resto de la militancia. El sistema político de género, ha producido algunos conceptos de enorme y constante utilidad por parte de los partidos políticos. Entre los que tenemos:

$\checkmark$ La línea partidista como argumento de verdad y autoridad

$\checkmark$ La pluralidad social y política como argumento erróneo de «integración» de las mujeres en los partidos. Las mujeres no somos un colectivo, somos la mitad del soberano. No debemos confundir pluralismo político con paridad o representación proporcional de género.

$\checkmark$ Una interpretación androcéntrica del perfil de cuadro político, al vaivén de lo que se cree demanda el «mercado electoral» para obtener el voto

$\checkmark$ El perfil y la promoción de cuadros políticos tomados del modelo masculino tradicional: blanco, buena presencia, corbata, señor casado, buen padre, buen ciudadano, etc.

$\checkmark$ En la formación de cuadros políticos están ausentes temas de formación en pensamiento feminista y perspectiva de género. Estos son considerados

\footnotetext{
${ }^{6}$ ARBOLEDA, María. Levantando el velo: Estudio sobre acoso y violencia política en contra de las mujeres autoridades públicas electas a nivel local en Ecuador. ONU, AMUME, AECID, Quito, 2012.
} 
como temas de mujeres y de poca importancia a otros más relevantes y de lo considerado como «coyuntura política».

$\checkmark$ Escaso y bajo presupuesto destinado a la formación de cuadros femeninos.

$\checkmark$ En la dirección de campañas encontramos mayormente hombres, con el tradicional perfil masculino del "estratega».

$\checkmark$ Las mujeres siguen desarrollando tareas feminizadas: secretarías, suplencias, etc., aunque, en muchos de los casos, representan más de la mitad de los y las afiliados y militantes

$\checkmark$ En la comunicación partidista y de campaña se mantiene una tipo de comunicación sexista, donde el cuerpo femenino es utilizado como una estrategia para captar votos y mejorar la imagen del partido o de campañas políticas;

$\checkmark$ La utilización de tonos desproporcionados o diferenciados al momento de dirigirse hacia las mujeres en asambleas partidistas; monopolización del uso de la palabra por los hombres;

$\checkmark$ En el ámbito de la planificación partidista es inexistente el diseño de planes de igualdad, y de acciones positivas que se planifiquen como acciones concretas para lograr los objetivos estratégicos de la organización. En la mayoría de casos vemos acciones positivas de formación pero que no están planificadas orgánica ni estratégicamente en los planes partidistas. Ej. Un ejemplo positivo los vemos en el II Plan de Igualdad del PSOE.

Para una mejor intervención en la lucha contra estos machismos sutiles o micromachismos, no es suficiente una definición limitada de violencia de género en cuanto espacio y actores. Se requiere de una versión más amplia e integral con alcance en la esfera pública de la política, y que lamentablemente no es reconocida en la mayoría de países que tienen legislación sobre violencia de género. Una de las definiciones un poco más amplia y compatible con el reconocimiento de los micro-machismos en la política, la encontramos en el CEDAW ${ }^{7}$, que no solo la limita la violencia de género a lo que ocurre en la esfera doméstica, del cónyuge o ex cónyuge, sino que la hace extensiva a las diferentes facetas sociales, políticas, económicas, etc. De la misma forma, tenemos lo dispuesto en la Ley autonómica de violencia de género de Cataluña Ley $5 / 2008^{8}$, la Declaración

7CEDAW, 1979. Convención sobre la eliminación de todas las formas de discriminación contra la mujer. "Parte I. Art. 1 A los efectos de la presente Convención, la expresión "discriminación contra la mujer" denotará toda distinción, exclusión o restricción basada en el sexo que tenga por objeto o resultado menoscabar o anular el reconocimiento, goce o ejercicio por la mujer, independientemente de su estado civil, sobre la base de la igualdad del hombre y la mujer, de los derechos humanos y las libertades fundamentales en las esferas política, económica, social, cultural y civil o en cualquier otra esfera." Disponible en: file:///C:/Users/J\%C3\%A9ssica\%20Elizabeth/Downloads/convencion_eliminacion_discriminacion_ceda w\%20(2).pdf (Consultado el 11/3/2014).

8 LEY 5/2008 de Cataluña, de 24 de abril, del derecho de las mujeres a erradicar la violencia machista. "Art. 3 A efectos de la presente ley, se entiende por: a) Violencia machista: la violencia que se ejerce contra las mujeres como manifestación de la discriminación y la situación de desigualdad en el marco de un sistema de relaciones de poder de los hombres sobre las mujeres y que, producida por medios físicos, económicos o psicológicos, incluidas las amenazas, intimidaciones y coacciones, tenga como resultado un daño o padecimiento físico, sexual o psicológico, tanto si se produce en el ámbito público como en el 
sobre la eliminación de la violencia contra la mujer, de la ONU de 1993, la Declaración de Beijing de $1995^{9}$, y la Convención Belem do Pará ${ }^{10}$.

Con posterioridad a los estudios diagnósticos realizados, Latinoamérica ha sido pionera en propuestas legislativas al respecto. El primer caso lo tenemos en Bolivia con la Ley de Acoso político y violencia política por razón de género que acaba de aprobarse. Igualmente es el caso de Ecuador, cuyo proyecto de ley fue presentado por AMUME, y sigue abierta su discusión. En México, desde finales del 2012, la Comisión de Igualdad de Género de la Cámara de Diputados aprobó incorporar en la Ley General de Acceso de las Mujeres a una Vida Libre de Violencia la modalidad de "violencia política de género", como una forma de trasgredir la integridad y los derechos de las mujeres en México. Este debate ya se ha extendido a otros estados y ciudades de la Unión como Chiapas ${ }^{11}$. Estas propuestas normativas surgen frente a las limitaciones normativas que han puesto al descubierto algunos problemas prácticos a la hora de combatir el acoso y violencia política en razón de género, como por ejemplo: la no existencia instancias públicas que realicen un monitoreo o seguimiento, verificación o registro de estos $\operatorname{casos}^{12}$.

Para terminar, podríamos decir que, en la aplicación de su autonomía y democracia interna, los partidos tienen una herramienta muy poderosa: la planificación orgánica interna, donde la perspectiva de género debe ser implementada de manera transversal, con aplicación del principio de paridad o presencia equilibrada no sólo en el momento de la confección de listas sino en la composición interna, en todos los niveles, espacios, funciones, órganos, etc., de las mujeres en toda su diversidad posibles: edad, territorio, cultura, orientación sexual etc., para evitar de esta manera también una posible discriminación múltiple. Todo esto no debe significar ninguna merma y menoscabo en el derecho de asociación de los partidos, y mucho menos en su libertad ideológica. No debemos olvidar, que los micromachismos en los partidos, al tratarse de una forma sui generis de violencia sutil, opera de manera estructural y simbólica, especialmente en el imaginario político de los partidos, de los actores políticos y del propio sistema democrático.

\footnotetext{
privado..." Disponible en: http://noticias.juridicas.com/base datos/CCAA/ca-15-2008.t1.html\#a (Consultado el 12/05/2014).

9 DECLARACION Y PLATAFORMA DE ACCION DE BEIJING. 1995. 113. "La expresión "violencia contra la mujer" se refiere a todo acto de violencia basado en el género que tiene como resultado posible o real un daño físico, sexual o psicológico, incluidas las amenazas, la coerción o la privación arbitraria de la libertad, ya sea que ocurra en la vida pública o en la privada..." p. 51. Disponible en: http://www.un.org/womenwatch/daw/beijing/pdf/BDPfA\%20S.pdf (Consultado el 11/04/2014).

10OEA. Art. 1 de la Convención Belén do Pará de 1994, la define como: “...cualquier acción o conducta, basada en su género, que cause muerte, daño o sufrimiento físico, sexual o psicológico a la mujer, tanto en el ámbito público como en el privado.." Disponible en: http://www.oas.org/es/cidh/mandato/Basicos/13.CONVENCION.BELEN\%20DO\%20PARA.pdf (Consultado el 2/2/2014).

${ }^{11}$ Nota de prensa. $\quad$ Disponible en:http://www.congresochiapas.gob.mx/pdf/iniciativas/INICIATIVA\%20DE\%20DECRETO\%20VIOLENCIA \%20POLITICA\%20POR\%20GENERO.pdf Disponible en: http://generalo.uimunicipalistas.org/mexico-reforman-leyes-para-visibilizar-violencia-politicaen-razon-de-genero/ ${ }^{12}$ ROJAS, María Eugenia. Acoso y Violencia Política...

Op. Cit. 2012.
} 


\title{
V. Conclusiones
}

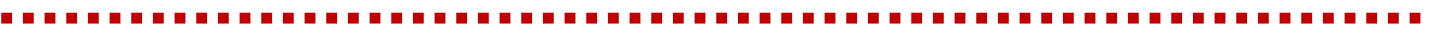

$\checkmark$ Es necesario tener en cuenta que, la lucha contra todo tipo de discriminación y violencia política por razones de género podría tener un impacto positivo en el fortalecimiento en la organización y funcionamiento de los partidos, y por tanto en el sistema democrático representativo en general.

$\checkmark$ Ante este contexto, es necesario profundizar la reflexión en torno a cómo los partidos deberían organizarse con estructuras igualitarias, mediante una planificación partidaria incluyente, lo que nos obliga a pensar en un concepto más operativo de igualdad política y partidista.

$\checkmark$ Debemos caminar no sólo hacia partidos con estructuras compuestas como lo dice LANCHESTER cuando se refiere a los partidos de la actualidad, sino con estructuras igualitarias.

$\checkmark$ El sistema político de género, donde los micromachismos aún se mantiene, es un problema que debe ser combatido si queremos alcanzar una sociedad auténticamente igualitaria, donde los partidos sean auténticas organizaciones democráticas con estructuradas internas igualitarias, y no todo lo contrario.
\end{abstract}

\section{Bibliografia}

ASOCIACIÓN DE MUJERES MUNICIPALISTAS DE ECUADOR. AMUME (2011). Proyecto de Ley de Acoso y violencia política por razones de género. Disponible en:

http://documentacion.asambleanacional.gob.ec/alfresco/d/d/workspace/Sp acesStore/80251378-7004-457f-91cb-

972b27613555/Ley\%200rg\%C3\%A1nica\%20contra\%20el\%20Discrimen,\%20 el\%20Acoso\%20y\%20la\%20Violencia\%20Pol\%C3\%ADtica\%20en\%20raz\%C3\% B3n\%20del\%20G\%C3\%A9nero (Consultado el 11/3/2014).

ARBOLEDA, María (2012). Levantando el velo: Estudio sobre acoso y violencia política en contra de las mujeres autoridades públicas electas a nivel local en Ecuador. Publicaciones de la ONU, AMUME, AECID, Quito.

ASTELARRA, Judith (2005). Veinte años de políticas de igualdad. Ediciones Catedra; Madrid.

BONINO, Luis (2004). "Los micromachismos». Revista La Cibeles № 2. Ayuntamiento de Madrid.

Convención sobre la eliminación de todas las formas de discriminación contra la mujer. CEDAW (1979). Disponible en: 
file:///C:/Users/J\%C3\%A9ssica\%20Elizabeth/Downloads/convencion_elimina cion discriminacion cedaw\%20(2).pdf (Consultado el 11/3/2014).

COBO, Rosa (1995). Fundamentos del patriarcado moderno: Jean Jacques Rousseau. Ediciones Catedra: Madrid.

DECLARACION Y PLATAFORMA DE ACCION DE BEIJING (1995). Disponible en: http://www.un.org/womenwatch/daw/beijing/pdf/BDPfA\%20S.pdf (Consultado el 11/04/2014).

FIGUERUELO BURRIEZA, Ángela (2007). «Representación política, derecho de asociación, y democracia Paritaria», Curso de Verano: Nuevas Tecnologías, administración y participación Ciudadana, Universidad de Salamanca. www.letrasjuridicas.com/Volúmenes/18/figueruelo18.pdf.

LANCHESTER, F (1996). «La propaganda elettorale (e referendaria) in Italia tra continuità sregolata e difficile rinnovamento», Quad. Cost., año XVI, nro. 3.

LEY 5/2008 de Cataluña, de 24 de abril, del derecho de las mujeres a erradicar la violencia machista. Disponible en: http://noticias.juridicas.com/base datos/CCAA/ca-15-2008.t1.html\#a (Consultado el 12/05/2014).

ORGANIZACIÓN DE ESTADOS AMERICANOS. OEA (1994). Convención Belén do Pará- Disponible en:

www.oas.org/es//13.CONVENCION.BELEN\%20DO\%20PARA.pdf (Consultado el $2 / 2 / 2014$ ).

ROJAS, María Eugenia (2012). Acoso y Violencia política en contra de Mujeres Autoridades públicas electas en los gobiernos locales-municipales en Bolivia. Publicaciones ONU-Mujeres, ACOBOL: Bolivia.

ROZA, Vivian (2010). Et. Al. Partidos políticos y paridad: La ecuación pendiente. Publicaciones IDEA internacional: Suecia, New York 
